\title{
Revealing the Urban Features of the Ancient Greek City of Mantineia through the Employment of Ground Penetrating Radar
}

\author{
A. Sarris, M. Manataki, C. Cuenca-Garcia, J. C. \\ Donati, T. Kalayci, \& N. Papadopoulos \\ Laboratory of Geophysical-Satellite Remote Sensing \& \\ Archaeo-environment (GeoSat ReSeArch Lab) \\ Institute for Mediterranean Studies, Foundation for \\ Research and Technology-Hellas (F.O.R.T.H.), \\ Nikiforou Foka 130, Rethymno 74100, Crete, Greece
}

Abstract-An extensive geophysical survey at the ancient Greek settlement of Mantineia in Peloponnese identified near surface features of archaeological interest that provide valuable information about the internal organization of the city. A multimethod approach was adopted for the application of various geophysical methods, spanning from magnetics to electromagnetics, soil resistance and GPR techniques, coupled with aerial and satellite remote sensing. The resolution of the GPR approach was crucial in identifying the details of the urban space and its development over time. The supremacy of the GPR in revealing the lateral expansion of the architectural residues and their vertical extent was obvious with respect to the rest of the methods applied.

Keywords- GPR, Greek settlements, Urban planning, Mantineia.

\section{INTRODUCTION}

Even though a number of ancient Greek settlements have been excavated in the past, most information about their urban planning is incomplete. This is the result of dispersed excavation trenches spread in various sections that give only a fragmented view [1]. In recent years, geophysical prospection techniques $[2,3]$, as well as satellite remote sensing [4], have been able to provide a more complete picture of the urban mechanics of cities in ancient Greece. These methods can scan large extents of the settlements and reveal a great deal of information about their spatial organization. This has become much more efficient nowadays with the use of multi-sensor instrumentation that can survey vast areas of archaeological sites. However, not all geophysical techniques have been successful in detecting structural remains. Even when some geophysical techniques are able to resolve large structures such as streets and city-blocks, they sometimes fail to identify other urban features. In contrast, the high resolution capabilities of the GPR technique have been successful in noting the details of city planning.

\author{
A. V. Karapanagiotou \\ Ephoreia of Antiquities of Arkadia \\ Hellenic Ministry of Culture, Education and Religion \\ Tripoli, Arkadia, Greece

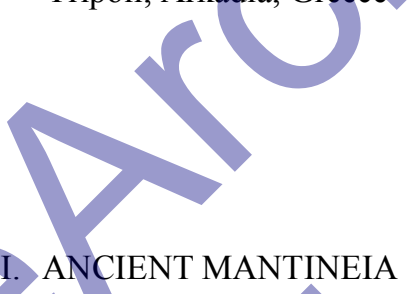

\section{ANCIENT MANTINEIA}

Mantineia was established sometime between 550-450 $\mathrm{BC}$ in the middle of the Arcadian plain in Peloponnese. The city was destroyed by the Spartans and the Macedonains on two separate occasions in the 4 th and 3 rd century BC, but it was rebuilt and played a prominent role in the region. The site, surrounded by a $4 \mathrm{~km}$ perimeter elliptical fortification wall, covers a total area of 120 hectares and remains wellpreserved with a number of public buildings (such as the agora and the theater). Excavations were initially carried out by the French School at Athens [5] and continued later on by Steinhauer [6] and more recently by the Archaeological Ephorate of Arkadia [1].

Previous small-scale geophysical work has been conducted at Mantineia using soil resistance and magnetic techniques [7]. More recently, the site was explored via a satellite remote sensing campaign employing a time sequence of various high-resolution satellite images and historical aerial photographs [4]. The processing of the imagery through the computation of spectral filters and vegetation indices identified a number of crop marks mainly related to the road grid system of the ancient city. The above methods provided the first indications that the ancient settlement consisted of an organized layout of N-S (spaced between $87-91 \mathrm{~m}$ ) and E-W (spaced between 59$60 \mathrm{~m}$ ) roads dividing the city into well-defined insulae (Fig. $1)$.

Despite the above methods and the past geophysical and archaeological excavations that pinpoint the existence of a planned settlement, the arrangement of the urban features of the site remained completely unexplored. 


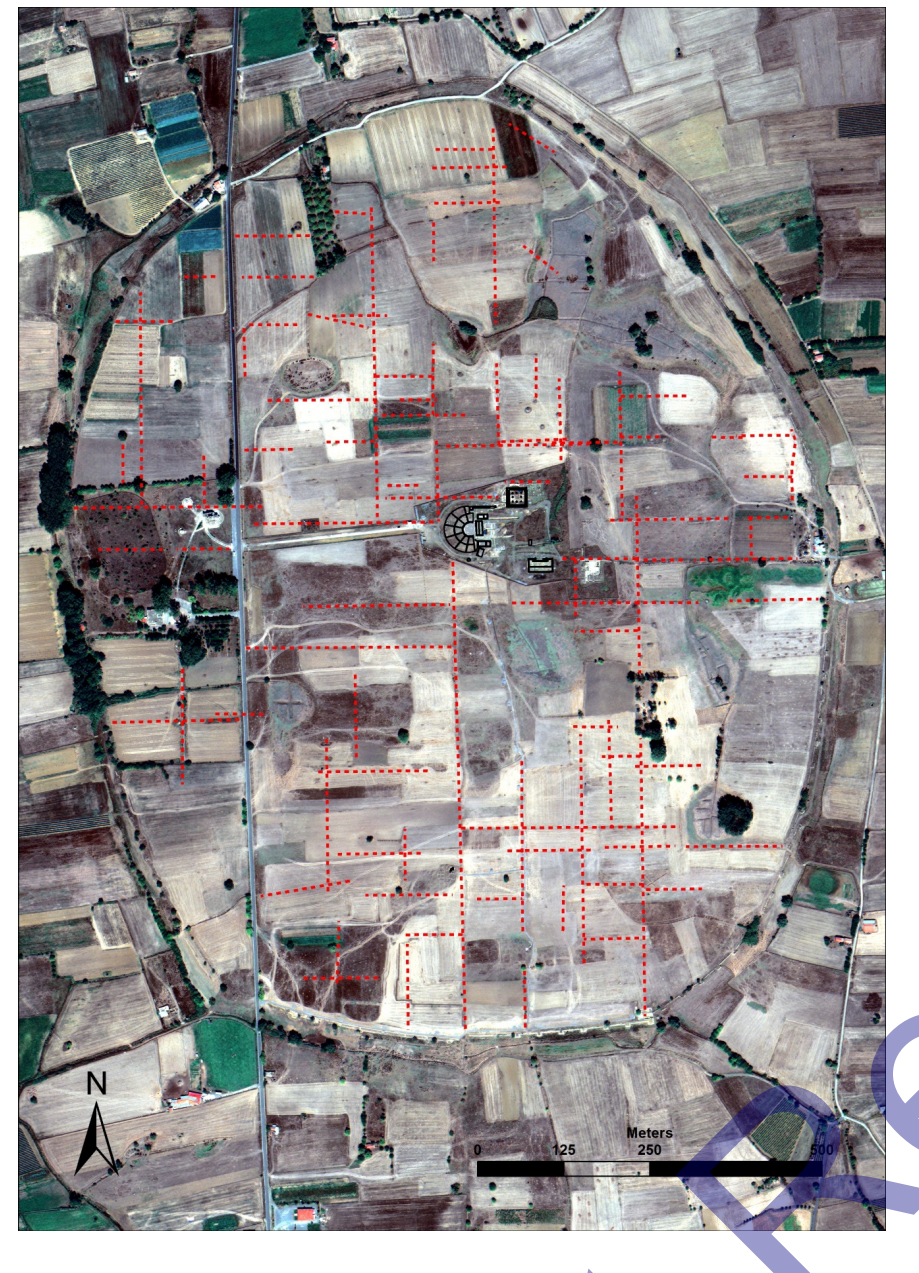

Fig. 1. Photo interpretation of soil and crop marks identified from a WorldView-2 image of Mantineia captured on 11 September 2013 brought to light a muber of linear features that reveal an organized layout of othogonal streets [4].

\section{METHODOLOGICAL TOOLS}

In the exploration of the ancient settlement of Mantineia, a single channel Sensors and Software NOGGIN Plus-Smart Cart system with a $250 \mathrm{MHz}$ antenna and a multichannel MALÅ Imaging Radar Array (MIRA) system consisting of nine $400 \mathrm{MHz}$ antennas were employed (Fig. 2). A total area of about 24,500 square meters was surveyed in sections of the site that were pinpointed through other datasets (especially magnetics and EM). The latter made use of a multi-sensor SENSYS magnetometer MX system consisting of 8 fluxgate gradiometers, a GEM-2 multi-frequency EM and a CMD multi-coil spacing EM device. Even though the coverage of the GPR was limited compared to the other methodologies (EM, magnetics and satellite remote sensing), in some cases it proved extremely useful as a tool of targeted resolution quality.

Despite the fact that the area is protected and only shallow cultivation is allowed, the raw GPR data had a high level of noise. Trace reposition, time zero correction (10-
$15 \%$ ), dewow filtering, SEC gain, background removal, bandpass filtering and migration were among the techniques used to process the data and enhance the GPR signals.
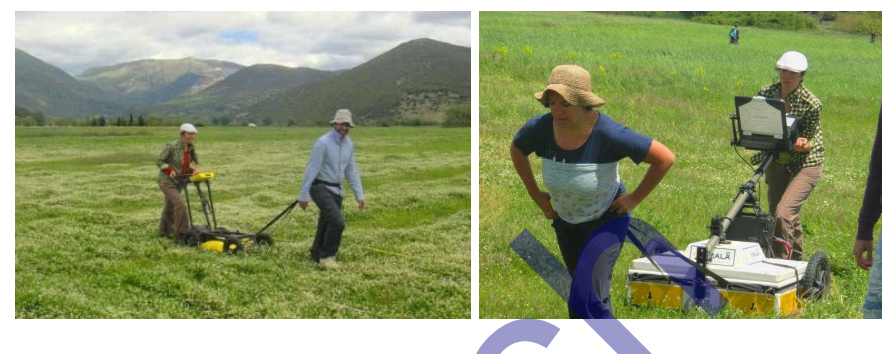

Fig. 2. Details of the employment of the single sensor Sensors and Software NOGGIN Plus-Smart Cart system with the $250 \mathrm{MHz}$ antenna and the multi-channel MALÅ Imaging Radar Array (MIRA) system at the archaeological site of Mantineia.

More precisely, for the processing of the data EKKO Project 2 and GFP from SENSORS \& SOFTWARE were used. The first step of the processing was related to the horizontal and vertical position of the including traces starting with trace reposition correction. This is a necessary correction when surveying into rough terrains where the starting and ending position of the survey lines is difficult to sustain the same. The correction is made by keeping the starting point of each line constant while adjusting the sampling step to meet the correct distance. For this to work properly, the occurring offset should be systematic. Next, the time-zero correction was applied to fix the vertical position of the first pulse that left the antenna and entered the subsurface. This correction trims the traces vertically by an amount that is expressed as a percentage of the first negative pulse and is consider as the first record in time. A Dewow filter was then applied to remove the low frequency noise related with the electrostatic and inductive fields near the antenna [Annan 2009]. At this point, SEC (Spreading \& Exponential Compensation) gain was applied to equalize the reflections that were recorded at deeper levels and appear weakened due to the signal's attenuation. Most times, besides the reflections, noise is also highlighted. In order to be removed without affecting the reflections, background average subtraction filter was used. The removal of the direct waves can also be consider as gain to highlight reflections from deeper levels since the former exhibit the highest amplitudes. Since noise was still present in the data, filtering in frequency domain was also used. A 1D bandpass filter was designed to retain frequencies at a certain range while removing the rest. The frequency range was selected from the radargramms average spectrum and it was focus near the antenna central frequency $(250 \mathrm{MHz})$. At this point, spatial coordinates were given to the radargramms according to the survey grid to create the subsurface volume. Then the instantaneous amplitude was estimated via Hilbert transform to better express the variations of signal's amplitude that delimits areas of high and low reflectivity. Additionally to instantaneous amplitude, migration was also used to convert the reflections from hyperbolas to point reflectors for a more realistic and accurate interpretation. 
Bandpass filtering and the application of SEC gain were particularly useful in removing the stripping effect of the plowing fields and emphasizing anomalies of small amplitudes located at greater depths. Time slices of increasing depth with a width of about $10 \mathrm{~cm}$ were also created in the final step to indicate the vertical extent of the underlying monuments.

\section{RESULTS}

The GPR survey targeted various zones of the city, including the area of the agora. Southwest of the agora, the GPR successfully mapped part of a residential block of the city and at the northeast corner of the agora the GPR results were also able to correct and complete the old French excavation plan.

More specifically, GPR at the southwest of the agora became informative as they clearly mapped a net of streets following a uniform alignment close to the cardinal points (Fig. 3). Furthermore, the results identified internal details of at least 3-4 buildings within a couple of city blocks. One of the structures appears to include a courtyard or a colonnaded room facing the west side of a N-S road. A few smaller compartments seem to be part of the complex in the back. The residues of an E-W road are obvious right to the west of the complex suggesting that the road predates the construction of the building. In general the city blocks along both sides of the $\mathrm{N}-\mathrm{S}$ road seem to be densely occupied by residential units.

Magnetic and GPR prospection was carried out to the east of the agora, in an area that has been partially excavated by the French in the $19^{\text {th }}$ century, but which has been reburied afterwards [5]. Both methods were successful in identifying a number of subsurface structures including public buildings and a long stoa consisting of a row of internal colonnades outlining the eastern side of the agora (Fig. 4). Even if the back wall of the stoa has been recently exposed by excavations, the geophysical methods, and especially the GPR, were successful in mapping the internal divisions of it, including its internal and external colonnades.

A couple of structures seem to extent next to the stoa towards the NE. Even if the particular structures are depicted in the French excavation plan, GPR data were able to provide much more detailed information about the particular structures. More precisely, the simple southern rectilinear $(23 \times 21 \mathrm{~m})$ structure turned out to consist of smaller rectilinear rooms around a central open space. The smaller rectilinear structure to the north has a different orientation from the one depicted on the French plan, and similar to another structure extended further to the NE, indicating different construction phases. The differences and similarities of the GPR results coming from the produced depth slices with the French plan are indicated on Fig. 4.

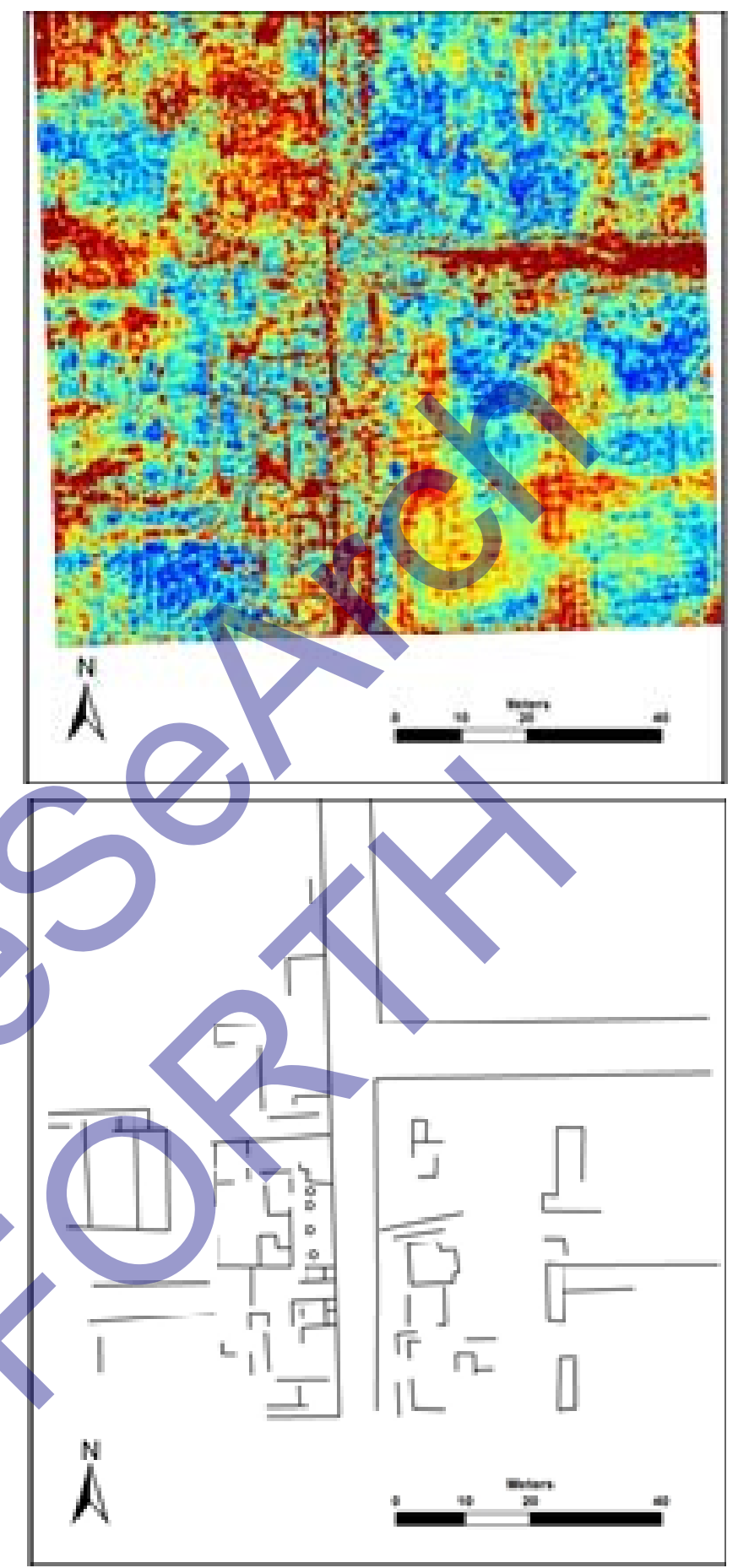

Fig. 3. Results (slice of $60-70 \mathrm{~cm})$ (TOP) and interpretation (BOTTOM) of the GPR results from the region southwest of the agora at Mantineia.

To the south edges of the fortification walls, depth slices (40-60cm) of the multi-channel MALA Imaging Radar Array (MIRA) system $(400 \mathrm{MHz})$ provided details of the region close to Gate H (Fig. 5). Both magnetic and GPR data indicated that occupation quarters were clustered at the center of the city leaving the vicinity of the fortification walls sparsely occupied. 

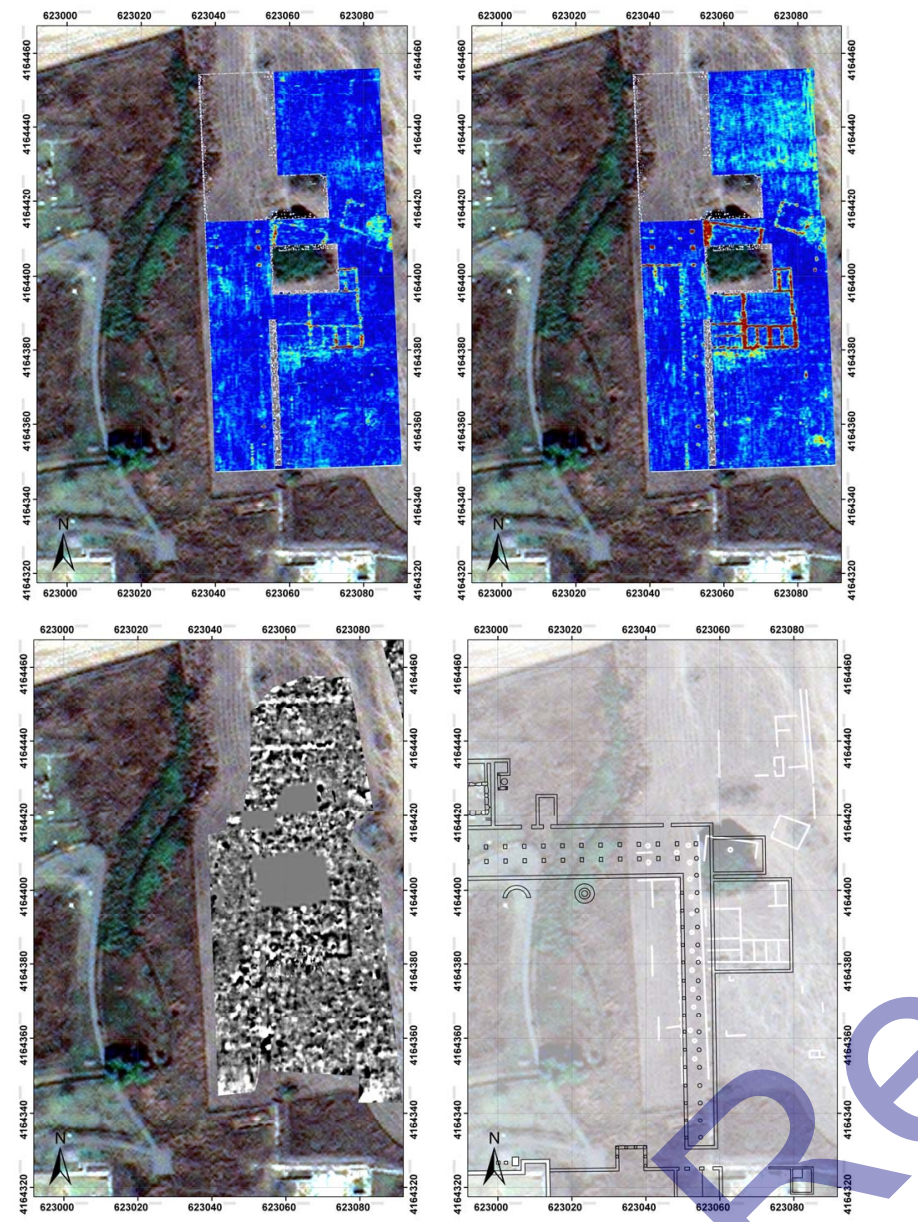

Fig. 4. Clockwise from top left: NOGGIN PLUS 250MHz depth slices for $60-70 \mathrm{~cm}$ and $100-110 \mathrm{~cm}$, Diagrammatic interpretation of the GPR signals taken from the eastern part of the agora, overlaid on the plan published in Mantinée et l'Arcadie Orientale [5]. Results of the SENSYS multisenor fluxgate gradiometer survey from the same region.

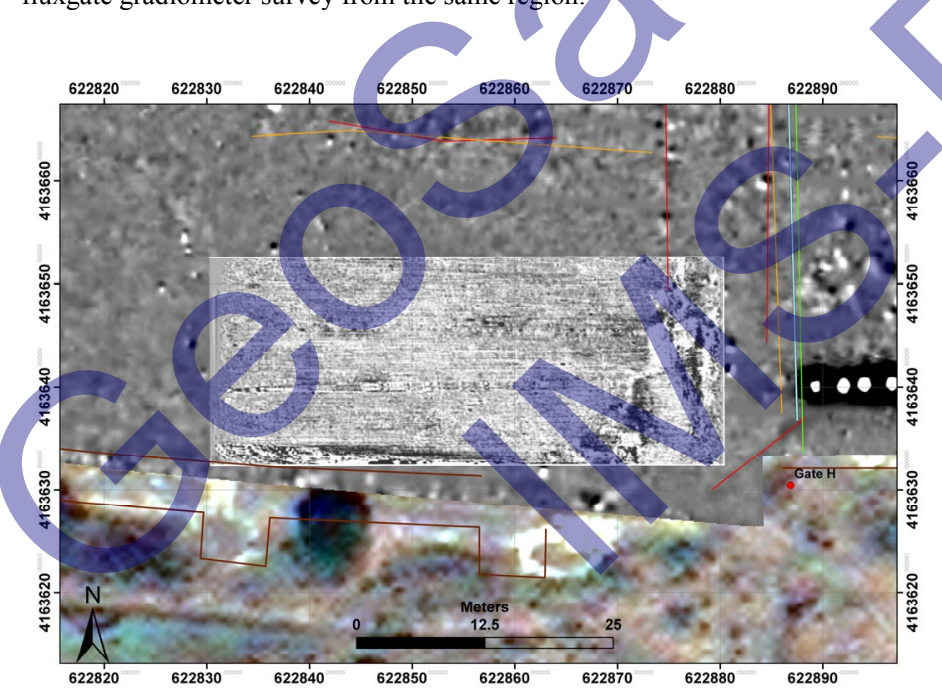

Fig. 5. Results (slice of $40-60 \mathrm{~cm}$ ) of the multi-channel MALA Imaging Radar Array (MIRA) system $(400 \mathrm{MHz})$ superimposed to the magnetic data from the southern part of the fortification walls of Mantineia (close to Gate $\mathrm{H})$.

\section{FINAL REMARKS}

The GPR survey targeted various sectors of the city, including the east of the agora where a stoa and even a row of internal colonnades were clearly outlined and the west of the agora where domestic unts were identifued in what it seems sto be an organized insalae of the city. Even if all the methods applied contributed to reveal the plan of the city of Mantineia, GPR was catalytic in providing internal details of the city's features. It became even more valuable with respect to the plans of old excavations and provided details of the internal organization of the habitation quarters and even the outer limits of the settlement.

\section{ACKNOWLEDGMENT}

This work was conducted in the framework of the POLITEIA research project, Action KRIPIS, project No MIS448300 (2013SE01380035) that was funded by the General Secretariat for Research and Technology, Ministry of Education, Greece and the European Regional Development Fund (Sectoral Operational Program: Competitiveness and Entrepreneurship, NSRF 2007-2013)/European Commission. The work has been carried out under the permit of the Hellenic Ministry of Culture (YППОА/Г $\triangle \mathrm{A} \Pi \mathrm{K} / \triangle \mathrm{BMA} / \mathrm{TE} \Xi E \mathrm{I} /$ $74653 / 45611 / 2457 / 181$ of $21 / 3 / 2014$ ) and of Dr. A. V. Karapanagiotou who is Head of the research program for the study, excavation and promotion of ancient Mantineia. The Ephoreia of Antiquities of Arkadia provided all the necessary material (topographic plans and excavation plans) from the research that is conducted in the area.

\section{REFERENCES}

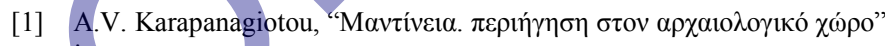
in press

[2] A. Sarris, N. Papadopoulos, V. Trigkas, E. Kokkinou, D. Alexakis, G. Kakoulaki, E. De Marco, E. Seferou, G. Shen, M. Karaoulis, K. Simirdanis, F. Kondili, M. Katifori, M. Dogan, Y. Lolos \& Th. Kalpaxis, "Recovering the urban network of ancient Sikyon through multi-component geophysical approaches", in A. Posluschn, K. Lambers \& I. Herzog (eds.), Layers of Perception. Proceedings of the 35th International Conference on Computer Applications and Quantitative Methods in Archaeology (CAA), Berlin, Germany, April 2-6, 2007. Kolloquien zur Vor und Frühgeschichte Vol. 10, Bonn 2008, pp. 11-16.

[3] A. Sarris, M. Teichmann, P. Seferou \& E. Kokkinou, "Investigation of the Urban-Suburban Center of Ancient Nikopolis (Greece) through the Employment of Geophysical Prospection Techniques", Computer Applications and Quantitative Methods in Archeology "Fusion of Cultures" CAA'2010, Fco. Javier Melero \& Pedro Cano (Editors), Granada, Spain, 2010.

[4] J. Donati \& A. Sarris, "Evidence for Two Planned Greek Settlements in the Peloponnese from Satellite Remote Sensing", American Journal of Archaeology, forthcoming 2016.

[5] G. Fougères, Mantinée et l'Arcadie Orientale. Paris: A. Fontemoing, 1898.

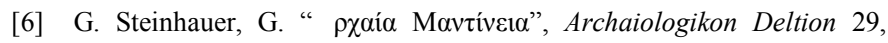
1973/1974, 296-301.

[7] Sarris, A. "Shallow Depth Geophysical Investigation Through the Application of Magnetic and Electric Resistance Techniques: An Evaluation Study of the Responses of Magnetic and Electric Resistance Techniques to Archaeogeophysical Prospection Surveys in Greece and Cyprus". A Ph.D. Dissertation, U. of Nebraska-Lincoln, Dept. of Physics and Astronomy, Lincoln, U.S.A. (U.M.I. Dissertation Services, A Bell \& Howell Company), 1992. 